\title{
Selected Methods of Measuring the Delay in Data Transmission Systems with Wireless Network Interfaces
}

\author{
T. Topor-Kaminski*, R. Żurkowski And M. Grygiel \\ Institute of Measurement Science, Electronics and Control, Silesian University of Technology, Gliwice, Poland \\ The article presents the concept of creating model of delays occurring in telecommunication system taking \\ into account all sources of delays generated during data transmission between the source and destination nodes. \\ Proposed model takes into account the existence of intermediary network devices that play a significant role in \\ the general delay budget. The paper presents two measurement systems used to measure delays in $802.11 \mathrm{~g}$ based \\ wireless networks and ones using ZigBee standard. Both systems were built based on the authors' concepts of \\ hardware and software allowing measurement of delays by using additional connection for the synchronization of \\ internal clocks. Described measurement systems selected amount of experiments was performed for varied network \\ structures.
}

PACS: 43.10.Pr, 89.20.Hh, 06.20.-f, 06.20.Dk, 07.05.Hd

\section{Introduction}

In the era of ongoing convergence of computing, measurement, control and communication systems end user becomes highly dependent on services offered by wide-area telecommunication systems. The range of services which include modern telecommunications networks range from traditional data services for individuals, to extensive control and measurement systems used in production environments.

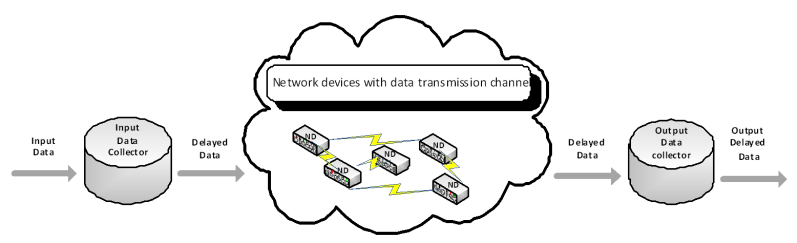

Fig. 1. A general model of the telecommunications system for one-way delay.

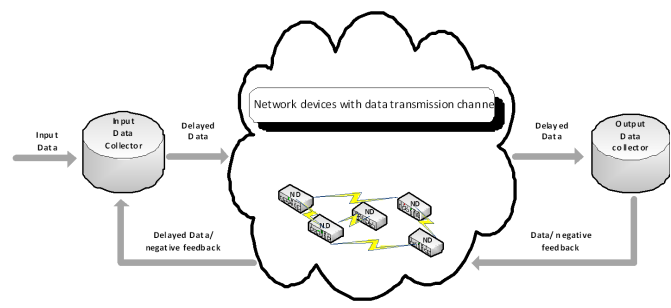

Fig. 2. A general model of the telecommunications system for delays in both directions.

\footnotetext{
* corresponding author; e-mail:

tadeusz.topor-Kaminski@polsl.pl
}

Long delays caused by low capacity of systems used in the production or control can cause major technical and physical problems [1-4] (incorrect test results, inaccurate control) which can affect result of the solution. In order to analyze the phenomena associated with delays in telecommunication systems should be adopted in its proper definition. It should be distinguished between two distinct types of delays whose analysis is somewhat different [5-7]. Namely, a delay in one direction is shown in Fig. 1 (one-way delay) and delay in both directions (two-way delay), Fig. 2.

Each of these kinds of delays is important for quite different types of transmitted data. A delay in one direction is connected with the transmission of data such as voice, video, etc. These are data streams which are analyzed in the output data collector (ODC), and have no impact on the further transmission. A delay in the two sides is associated mainly with all kinds of automation systems - measuring and control systems [1]. In such systems, there is usually an object called a regulator and regulated mostly inert object between which the exchange of data with the use of feedback. This exchange takes place through a telecommunications system. At the same time, it should be noted here that due to the nature of the telecommunications system in our case the delay in one direction for the same system is always different from the delay in the opposite direction or delay in the two sides is twice the value of the delay in one direction [5-8]. Given the above considerations, the following definitions are: one-way delay is called the time between the introduction, preparation of data by input data collector (IDC), and the moment in which the information is received and prepared for use by ODC, the delay in both sides is called the time between the introduction, preparation of data by IDC, and the moment in which the information is received and again prepared for use by IDC after its receipt, preparation, processing and retransmission by the ODC. Delays can be influenced by many factors [6], which can 
be described both deterministic and probabilistic. Using deterministic dependences is usually determined by the maximum delay in networks [8], while the probabilistic measures are used to obtain distributions of delays resulting from the factors described random. Probabilistic description of the delay provides full information about their properties [9], and therefore can be used to analyze the network properties much wider than the deterministic description.

Let us consider the simplest case, when the IDC and ODC are members of computer network and communicate with each other without using any other devices. Such a situation is shown schematically in Fig. 3.

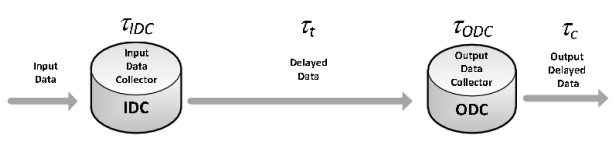

Fig. 3. Sources of delays in the transmission from IDC to ODC.

$\tau_{\text {IDC }}$ is a symbol denoting the time required to prepare the data and gain access to the media (communication medium) in the IDC, and $\tau_{\mathrm{t}}$ as the time required to send the message from the IDC to the ODC, $\tau_{\text {ODC }}$ as a time of receipt and preparation of data in the ODC. Assuming that the transmission is initiated at the time labeled $t_{0}$, and the start of transferring data over the network occurs at the time $t_{\mathrm{IDC}}$, the transmission delay associated with IDC is equal

$$
\tau_{\text {IDC }}=t_{\text {IDC }}-t_{0} .
$$

The delay $\tau_{\mathrm{t}}$ of transmitting the message from node IDC to node ODC is the difference between the moment $t_{\mathrm{t}}$ and the moment of initiation of transmission $t_{0}$. This delay, given the dependence of (1), is equal

$$
\tau_{\mathrm{ODC}}=t_{\mathrm{t}}-t_{0}=\tau_{\mathrm{t}}+t_{\mathrm{IDC}}-t_{0}=\tau_{\mathrm{t}}+\tau_{\mathrm{IDC}},
$$

which is the sum of two partial delays involved. Total delay in this case is equal

$$
\begin{aligned}
\tau_{\mathrm{c}} & =t_{\mathrm{ODC}}-t_{0}=\tau_{\mathrm{ODC}}+\tau_{\mathrm{t}}+t_{\mathrm{IDC}}-t_{0} \\
& =\tau_{\mathrm{IDC}}+\tau_{\mathrm{t}}+\tau_{\mathrm{IDC}},
\end{aligned}
$$

where $t_{\mathrm{ODC}}$ is time upon completion of data collection and preparation of the ODC. Thus, the total delay is the sum of delays stemming from the partial individual elements involved in the data transmission.

\section{Transmission delays models}

Based on the definition proposed in the introduction, you can build a model of delays in data transmission (Fig. 4). This model includes all sources of delays that may occur in the telecommunication system. It is a theoretical model that was developed in order to facilitate analysis of the phenomena affecting the delays during data transmission via computer network.

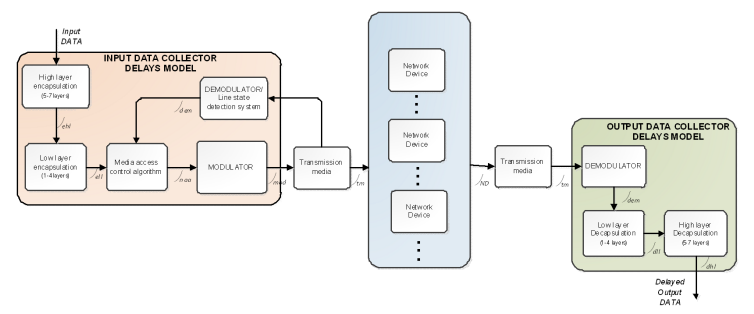

Fig. 4. Model of data transmission delays where $\tau_{\text {ehl }}, \tau_{\text {ell }}$ denote the delay introduced by the encapsulation process of the upper and lower layers of the open source interconnection (OSI) model, $\tau_{\text {naa }}$ is the delay generated by the media access control algorithm, $\tau_{\text {mod }}$ means the delay generated by the modulation process, $\tau_{\text {dem }}$ means the delay generated by the demodulation process, $\tau_{\mathrm{tm}}$ is the delay introduced by the transmission medium, $\tau_{\mathrm{ND}}$ is the delay introduced by the network device, $\tau_{\mathrm{dll}}, \tau_{\mathrm{dhl}}$ denote the delay introduced by the reverse encapsulation process of lower and higher layers of the OSI model.

In the model shown at Fig. 4, we can distinguish three main blocks namely: IDC delay model, the ND model delays (network device) and a late model ODC. Between the above-mentioned blocks is suitable transmission medium which generates delay characteristic of its nature.

IDC delay model is characterized by the actions you must take to prepare and transmit data via the transmission medium. The first element of a model called the high encapsulation layer corresponds to delays associated with the actions represented by the top three layers of the OSI model [5, 6] (application, presentation, session). These layers are primarily responsible for the correct coding, presentation of data and for compiling and maintaining a session between the IDC and ODC. Another element of low layer encapsulation represents 1-4 layers of the OSI model (transport, network, data link and physical). Transport and network layers are characterized by the relevant protocols adopted during the transmission while today they are dominated by pairs of transmission control protocol/Internet protocol (TCP/IP) and (user datagram protocol/Internet protocol (UDP/IP).

Separation of layers in the model above the lower is justified by the fact that the actions of higher layers are carried out mostly at the level of application software while the lower classes are usually associated with the operating system and hardware systems such as medium access network interface card (NIC) [5, 6].

Further elements of the model are associated with medium access layer (MAC) and physical layer (PHY) of the OSI model. The first one media access control is part of the program algorithm working in close cooperation with modulator's and demodulator's hardware. It is usually represented by two algorithms: carrier sense multiple access with collision detection (CSMA/CD) which is the carrier detection algorithm with multiple access and collision detection and is characteristic for the wired Ethernet. Carrier sense multiple access with collision 
avoidance (CSMA/CA), like its predecessor, is the carrier detection algorithm but with multiple access collision avoidance, and is characteristic for a wireless Ethernet technology. Modulator and demodulator circuits are usually specialized integrated circuits responsible for the conversion of data to a signal suitable for the transmission medium that is: an electrical signal, optical signal or a signal in the form of electromagnetic wave.

Given the delays associated with the above-described elements, the total delay generated by the IDC can be marked as $\tau_{\text {IDC }}$ present as the sum of delays introduced by each of the elements of the model of Fig. 4 according to the relation

$$
\tau_{\text {IDC }}=\tau_{\text {ehl }}+\tau_{\text {ell }}+\tau_{\text {naa }}+\tau_{\text {dem }}+\tau_{\text {mod }} .
$$

Data addressed in the proposed model in Fig. 4 is an object called ODC. It represents both of these actions to be taken to restore the data that comes to its input with the transmission medium. The first essential element in the chain of ODC activity is a physical signal demodulator which converts the information into digital form. Then there are two elements inducing the delay and related with reverse encapsulation process, namely: low layer and high layer reverse encapsulation. They perform the reverse process for the encapsulation process described above. The process for the same reasons as for the IDC has been divided into two parts associated with higher and lower layers of the OSI model. Given the foregoing, the delay induced by the ODC on the lines of Fig. 5 is defined by the relation:

$$
\tau_{\text {ODC }}=\tau_{\text {dem }}+\tau_{\text {dll }}+\tau_{\text {dhl }} .
$$

Between IDC and ODC in a particular case there are only suitable transmission media. In fact, we are dealing with the situation when the data must travel a longer distance usually by various types of transmission media. It is necessary in this case, to use of appropriate equipment intermediary, in the present situation are the parts marked network devices. We can distinguish many types of network devices whereby one can extract the device main groups namely: switches, routers, firewalls or UTM devices and proxy servers. The role and functions of each group of devices are quite different. They operate on different layers of the OSI model, performing different functions. However, you can specify the overall structure that reflects the source of delays for each network devices. Figure 5 presents the universal model for network delay devices.

The model of Fig. 5 contains all the elements that are common to all types of network devices and are a source of delays in data transmission. The first part of the model is the demodulator performing the same function as for the ODC. Next process reverse to encapsulation must be used but it will cover the different layers of the model axis, depending on the type of device. For example for switch there will be a layer 1-2, 1-4 for the router, the device UTM 1-7. The role of the next element is called the input buffer which memorize and store data about

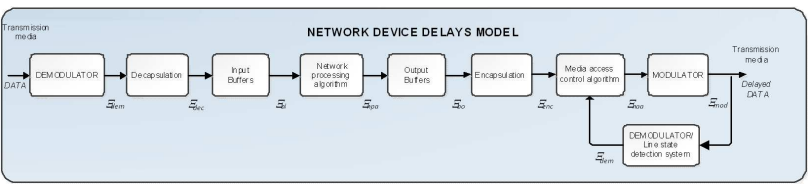

Fig. 5. Network device delay model where $\tau_{\text {dem }}$ means the delay generated by the demodulation process, $\tau_{\text {dec }}$ means the delay generated by the reverse encapsulation process, $\tau_{\text {npa }}$ means the delay generated by the network processing algorithm, $\tau_{\text {bi }}, \tau_{\text {bo }}$ denote the delays associated with input and output buffers, $\tau_{\text {enc }}$ means the delay generated by the process of encapsulation, $\tau_{\text {naa }}$ means the delay generated by the media access control algorithm, $\tau_{\text {mod }}$ means the delay generated by the modulation process.

the appropriate length for processing algorithm. Network processing algorithms performs tasks specific to a particular group of devices such as the switches performs packet switching functions, routers are responsible for routing of packets to the appropriate node, firewalls performs the functions required in filtration of packages. Then there is the element storing data gathered by the algorithm that processes collected data - output buffer. Another model elements represent the actions required in preparation of data to send or encapsulation process suitable for the type of device layers of the model axis and then the process of modulation and transmission using appropriate media access algorithm, similarly as it does for IDC. Given the model of Fig. 5, the delay introduced by the network devices can be written in the form of dependences given below

$$
\begin{aligned}
& \tau_{\mathrm{ND}}=\tau_{\mathrm{dem}}+\tau_{\mathrm{dec}}+\tau_{\mathrm{bi}}+\tau_{\text {npa }}+\tau_{\mathrm{bo}}+\tau_{\mathrm{enc}}+\tau_{\text {naa }} \\
& \quad+\tau_{\text {dem }}+\tau_{\text {mod }} .
\end{aligned}
$$

Specifying in the model all the elements that generate delays in the telecommunication system can determine the total delay (complete). The total delay associated with the transmission of data between the IDC and ODC in accordance with the proposed model is the sum of the delays caused by all the elements contained in the model of Fig. 4 and expressed the relation

$$
\tau_{\mathrm{COM}}=\tau_{\mathrm{IDC}}+\tau_{\mathrm{ODC}}+\tau_{\mathrm{ND}}+\tau_{\mathrm{tm}} .
$$

The proposed model of the system delays of the transmission of data allows the analysis of phenomena associated with each individual source of delays in the transmission system. This analysis facilitates the construction of equipment for measuring the delay of data transmission in computer networks.

\section{Wireless media transmission delay measurement system}

Based on the model presented in the second section a data transmission delay meter had been built. Such a device can be built based on appropriate software and a PC equipped with two network cards, but this measure- 
ment will be burdened with many errors, sourced mainly by the operating system. Therefore, there is a need to build a meter which with simplified software and hardware solutions used, will eliminate these errors.

Built data transmission delay meter consists of two interconnected systems, the transmitter and receiver, Fig. 6. In both uses mini modules MMnet104 [9, 10] built on the ATmega128 microcontroller [11] and Ethernet interfaces LAN91C111 [12] were used. Block diagram of the data transmission delay meter is shown in Fig. 7.

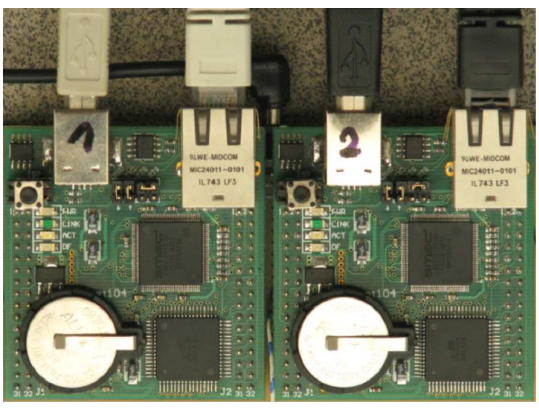

Fig. 6. Meter transmission delays.

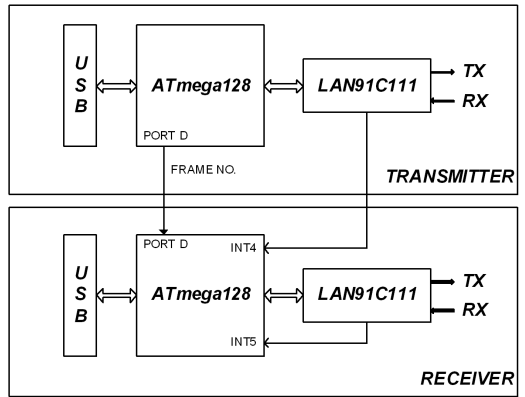

Fig. 7. Block diagram of the transmission delays meter.

The task of the microcontroller's software in the transmitter is to prepare and send to the Ethernet interface of the data packets of a certain length at specified intervals. For each transmitted data, packet transmitted to the receiver transmitter 4-bit number, and a signal indicating the correct transmission. Diagram of the transmitter in the microcontroller is shown in Fig. 8. In the software encoder there is implemented the support for ARP and IP protocols (ICMP and UDP) [13]. Use of USB interface allows you to change the length of transmitted packets, their number and IP addresses without having to reprogram the microcontroller.

The receiver stores it in a temporary marker until the end of the array of data transmission, together with assigned to it by the transmitter number. This number helps to identify where the packet transmission time is longer than the interval between broadcasts of data packets. At the moment when the receiver reaches the correct data packet, the timestamp is stored in its pickup. The next step is to analyze the contents of the data packet.

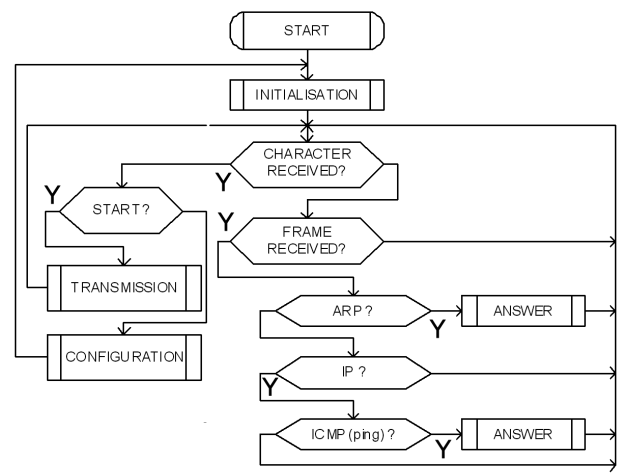

Fig. 8. Diagram of the transmitter.

If the received packet originates from the transmitter, which is identified on the basis of data fields of UDP frame, then a temporary array is read with the corresponding time stamp of origin and stored along with the time of receiving the package and its number in results array. An array with the results of measurements is successively transmitted through the USB interface to a computer, processed, and the results saved as a text file containing the results of the measurement data packet transmission times. Diagram of the program in the microcontroller of the receiver is shown in Fig. 9. In order to measure the transmission time, receiver's microcontroller is used.

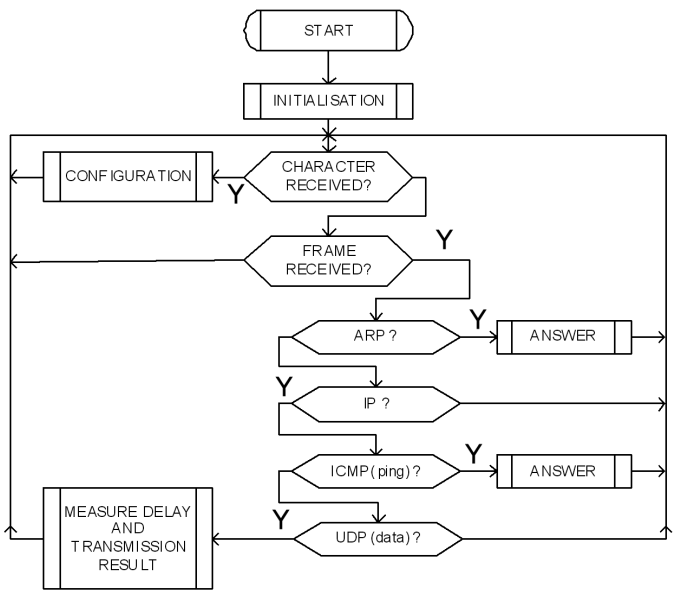

Fig. 9. Diagram of the receiver.

Analysis of the received packet data is divided into several stages:

- download the entire packet of data from system memory interface to the microcontroller;

- read the Ethernet header protocol information,

- choose the appropriate protocol for handling subroutine. 
The software module of the receiver detects two protocols: ARP, and IP. If the data is sent from another protocol, they are ignored. If the ARP request is received then the module sends back the answer, allowing switches and routers in the network to identify the port to which packets are supposed to be sent next time.

For IP protocol support are two upper layer protocols, they are ICMP — which is implemented support for "ping" and UDP port through which there is communication with the module. If the received data packet in the UDP data field will be the string "Datagram", this is an indication that the received packet comes from the transmitter, and the next two bytes contain the number. The youngest 4 bits number addresses the package to a temporary array which is read by the package to give a time stamp, and data are stored in the scoreboard.

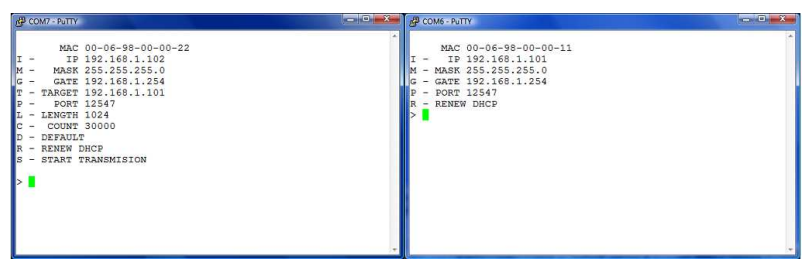

Fig. 10. Configuration mode (a) transmitter, (b) receiver.

To handle the delay meter, a computer program is used for the serial terminal. Figure 10 shows views of the terminal window during the configuration of the transmitter's and receiver's parameters. Registration of the measurement results is also performed in the terminal using the function of the log recorder. For the delay measurement there is used IP/UDP, in which there is no automatic retransmission of erroneous data packets. Thus based on the amount of packets not transmitted the reliability of links is determined (grated packages).

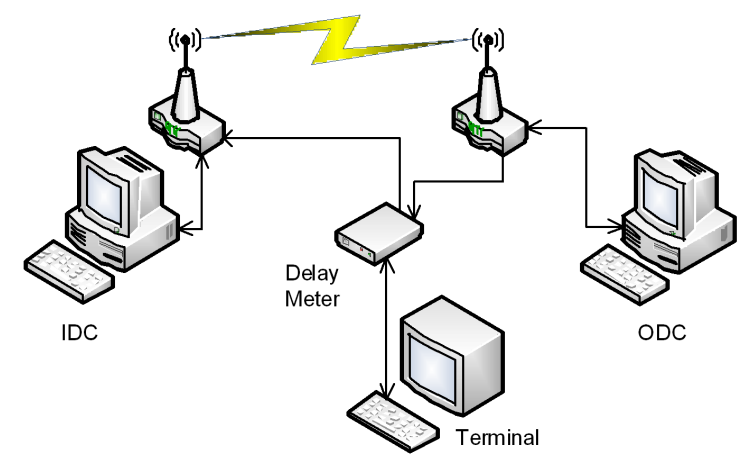

Fig. 11. Schematic of the measuring system.

Performing measurements of the meter is connected to delay elements of the network infrastructure as shown in Fig. 11.

Gauge delays combined with two access points tested were equipped with network switches. A single series of measurements consists of 30,000 packets transmission of length. In the measurements presented in this paper, measurement of impartiality is not included, but in subsequent studies, the expansion of the measuring system and this parameter will be determined. The system uses wireless access points, the Linksys WRT54G, which changed the original software, the alternative (recommended by the manufacturer) software Tomato. Results of measurements are summarized in Table and a sample histogram is shown in Fig. 12.

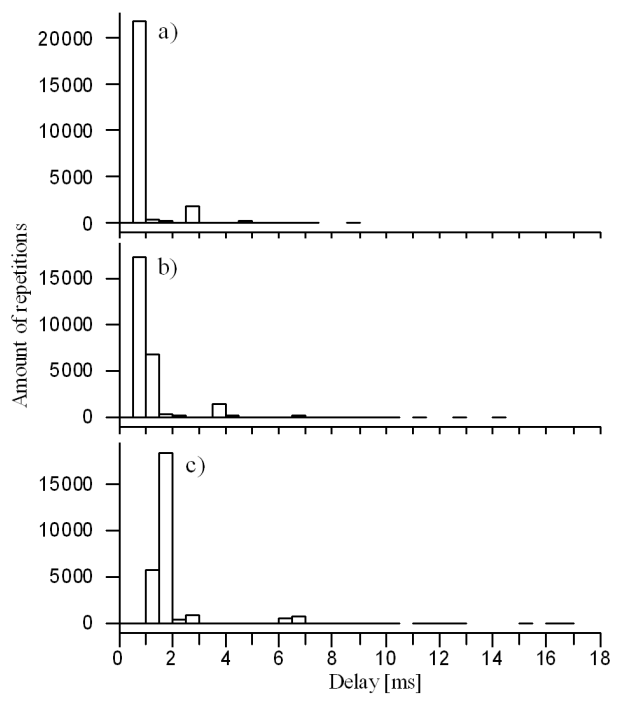

Fig. 12. Typical histograms of the transmission delays for data packets of different lengths: (a) 1, (b) 512 , (c) 1472 bytes.

TABLE

Summary of results of measurements of delays.

\begin{tabular}{c|c|c|c|c|c}
\hline \hline Conditions & $\begin{array}{c}\text { Packet } \\
\text { size }\end{array}$ & Delay & $\begin{array}{c}\text { Max } \\
\text { delay }\end{array}$ & $\begin{array}{c}\text { Delay } \\
\text { fluct. }\end{array}$ & $\begin{array}{c}\text { Packet } \\
\text { loss }\end{array}$ \\
\hline Without & 1 & 0.8 & 10.8 & 10.1 & 16 \\
coding & 128 & 0.9 & 13.7 & 12.8 & 13 \\
& 512 & 1.1 & 16.4 & 15.2 & 11 \\
& 1024 & 1.4 & 22.0 & 20.5 & 10 \\
& 1472 & 1.6 & 21.3 & 19.7 & 8 \\
\hline Without & 1 & 0.8 & 10.8 & 10 & 17 \\
coding & 128 & 0.9 & 10.8 & 9.9 & 12 \\
+ ping & 512 & 1.1 & 14.0 & 12.9 & 10 \\
between & 1024 & 1.4 & 18.3 & 16.9 & 10 \\
computers & 1472 & 1.6 & 16.8 & 15.1 & 8 \\
\hline Coding & 1 & 0.9 & 8.7 & 7.8 & 18 \\
WPA & 128 & 1.1 & 14.9 & 13.8 & 12 \\
TKIP & 512 & 1.3 & 14.4 & 13.1 & 11 \\
& 1024 & 1.7 & 18.3 & 16.6 & 11 \\
& 1472 & 1.9 & 27.3 & 25.4 & 9
\end{tabular}




\section{Measurement of data transmission in the zigbee delays based networks}

Telecommunication systems with specific properties are wireless sensor systems. In such systems, one of the best working standards is the IEEE 802.15.4 [14, 15]. This standard is designed for sensor networks, telemetry, and measurement and control. It defines the communication with the relatively low speeds, while allowing to simplify the transmission modules. This standard defines the physical layer describing the low-rate wireless personal area network (LR-WPAN). Higher layer functions are implemented in accordance with the ZigBee specification [16]. For the above-mentioned communication standard according to the previously described model, a measuring system has been developed allowing measuring the delays occurring in data transmission in ZigBee networks. Implementation of such system is possible in networks built of modules that manufacturers made it possible to communicate through a serial interface. Measurement of data transmission delay in one direction has been made in the network operating in a star configuration and the configuration of the tree [15-19].

Wireless network used to measure transmission delays was built based on the AUREL company modules. Wireless modules are equipped with serial interface. This allows the wired observations of incoming and outgoing data by radio link.

In the study, the emphasis is on checking the influence of network configuration occurring in the data transmission delays. Delays were examined for two different network configurations. Diagram of the network working in a star configuration is shown in Fig. 13. Diagram of the network working in the tree configuration is shown in Fig. 14.

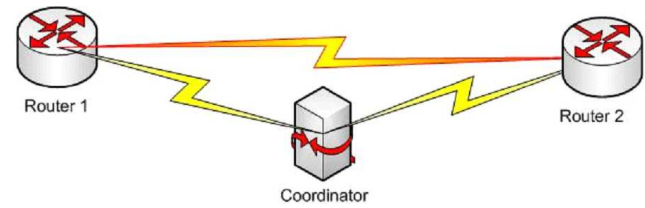

Fig. 13. Measuring system in star topology.

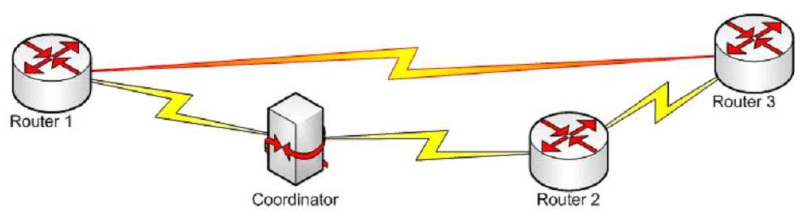

Fig. 14. Measuring system in tree topology.

In the star configuration, routers that are both appropriate transmitter and receiver were connected directly with the system coordinator. However, in the configuration shown in Fig. 14 data communication between a router 1 and the router 3 mediated second router.
The network was controlled by the network coordinator, which was under control of software installed on PC.

Due to the fact that many manufacturers of ZigBee based network components enable serial data transfer, there was the concept of measuring delays in these networks based on the difference in time required to send and receive serial data. For the realization of the measurement of delays in data transmission, a measurement system was built. The block diagram is shown in Fig. 15.

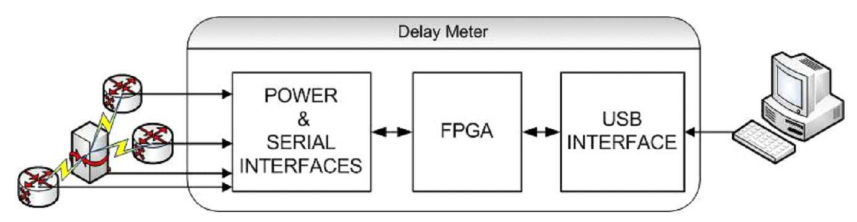

Fig. 15. ZigBee delay measuring system.

The main element of the system is a delay meter, thus allowing to measure latency in data transmission in ZigBee based networks. Based on functionality, the meter of data transmission is composed of three elements. The first is the power supply and interface system, which allows connecting components of the tested ZigBee network. Developed in LabView supervision software was designed, which allows communicating with the delay meter using USB interface. The final element is a programmable FPGA logic circuit in which the conversion is carried out and timing of transmitted messages.

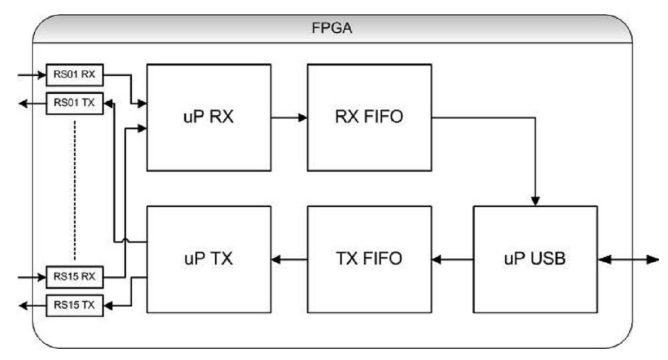

Fig. 16. FPGA internal structure.

Inside the FPGA structure 15 complete asynchronous serial interfaces were implemented, designated in the block diagram of Fig. 16 as RSxx symbols RX and TX RSxx. The structure also includes three Picoblaze microprocessors and two FIFO memories, corresponding in communication between the processors. Software running on microprocessor uP RX checks whether any of the serial ports received sent byte in which case it sends it to the RX FIFO memory by creating a frame that contains the serial interface number of the byte originates, the timestamp of its occurrence and the received byte $\mathrm{uP}$ TX task is performed by reading TX FIFO memory frame containing the destination port number and then sending it to the correct serial port. The last chip is USB uP, whose task is to exchange data between USB interface and FIFO memory. 
The measurement of latency is carried out in PC based on the difference of timestamps of bytes received from individual serial ports.

Measurements were made for transmission of messages with a length of 10 bytes in the network configurations shown in Fig. 13 and Fig. 14. The measurement results are summarized in the form of histograms in Fig. 17a and b.

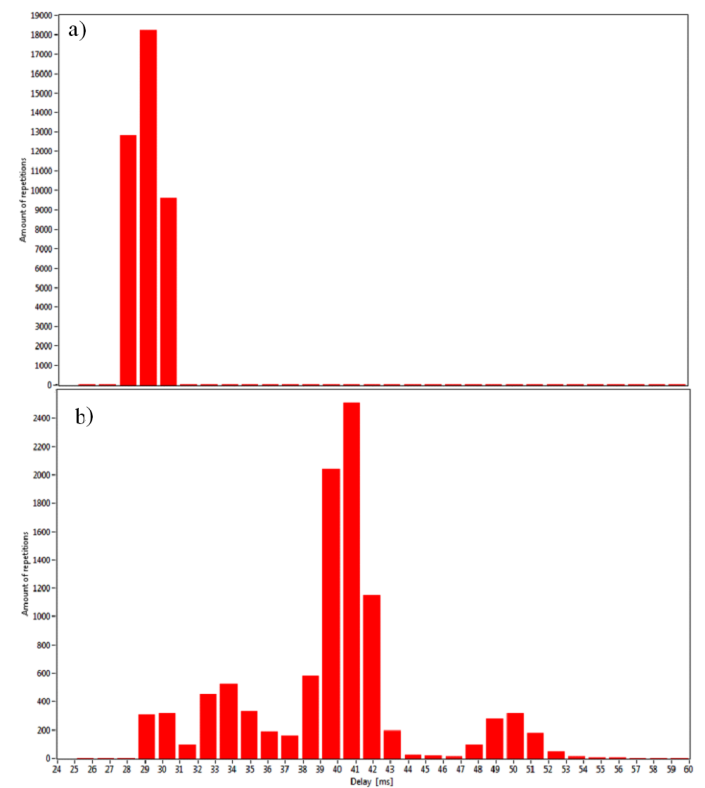

Fig. 17. Delay histogram for: (a) star topology, (b) tree topology.

\section{Conclusions}

The paper presents concepts for the implementation of systems allowing measurement of delays in transmission of data occurring in telecommunication systems, ranging from theoretical assumptions or models to the physical realization in the form of measuring devices. The idea of measuring delays is not as trivial matter as it may seem, and often as shown in the article requires an individual approach to the telecommunications standard. Another problem is the complexity of the topology of the network. Considerations presented in the paper are one of the first stages of research on the comprehensive analysis of delays encountered in telecommunication networks. Authors are aware that experiments conducted from the standpoint of measuring the actual transmission systems are trivial, but it allows verification of theoretical assumptions and foundations for further research. Upon completion of the article the work on the future versions of the measurement systems that are more powerful and adapted to perform measurements in more universal environment are in progress. Another area of research being undertaken by the authors is to seek a mathematical apparatus which allows one describing the phenomena associated with delays in a probabilistic manner. Some basics of this camera can be found in [9], however, it requires adaptation to the proposed working model.

\section{References}

[1] P. Kielan, T. Trawiński, Przeglad Elektrotechniczny (Electrical Review) 86, 86 (2010) (in Polish).

[2] T. Pustelny, C. Tyszkiewicz, K. Barczak, Opt. Appl. 33, 469 (2003).

[3] K. Barczak, T. Pustelny, D. Dorosz, J. Dorosz, Europ. Phys. J. Special Topics 154, 11 (2008).

[4] T. Hejczyk, M. Urbanczyk, W. Jakubik, Acta Phys. Pol. A 118, 1148 (2010).

[5] L. Larry, R. Peterson, S. Bruce, I. Dave, Computer Networks - A System Approach, 5th ed., Morgan Kaufmann, 2011.

[6] S. Andru, J. Tanenbaum, J. David, T. Wetherall, Computer Networks, 5th ed., Pearson, 2011.

[7] M. Hassan, R. Jain, High Performance TCP/IP Networking: Concepts, Issues, and Solutions, Prentice-Hall, 2003.

[8] E. Michta, Communications Models of MeasurementControl Network Systems, Vol. 38, Zielona Góra Technical University, Zielona Góra 2000 (in Polish).

[9] J. Jakubiec, Errors and Data Uncertainties in Measurement-Control Systems, Vol. 76, Silesian University of Technology, Gliwice 2010 (in Polish).

[10] www.propox.com .

[11] www.atmel.com .

[12] www.smsc.com .

[13] J. Bentham, TCP/IP Lean - Web Servers for Embedded Systems, CMP, Wiley, London 2000.

[14] T. Topór-Kamiński, R. Żurkowski, in: Proc. VIIIth Conf. on Measurement Systems in Technical Science and Industry, Lagow (Poland), Zielona Góra Technical University, Zielona Góra 2010, p. 171 (in Polish).

[15] 802.15.4 IEEE Standard for Information Technology - Telecommunications and Information Exchange between Systems - Local and Metropolitan Area Networks - Specific Requirements. Part 15.4: Wireless Medium Access Control (MAC) and Physical Layer (PHY) Specifications for Low-Rate Wireless Personal Area Networks (LR-WPAN), New York 2003.

[16] ZigBee Specification v1.0 ZigBee Document 053473r00, ZigBee Alliance, San Ramon 2005.

[17] M. Grygiel, R. Żurkowski, Experimental system for measuring latency in ZigBee 802.15.4 standard, Wydział Elektrotechniki i Automatyki, Politechnika Gdańska, Gdańsk 2009 (in Polish).

[18] Guide to the Expression of Uncertainty in Measurement, ISO/IEC/OIML/BIPM, 1992 (1995).

[19] P. Struk,T. Pustelny, K. Gut, K. Gołaszewska, E. Kamińska, M. Ekielski, I. Pasternak, E. Łusakowska, A. Piotrowska, Acta Phys. Pol. A 116, 414 (2009). 